Letter to the Editor.

Carlos Corredor.

\title{
A problematic enzyme
}

Dr Fell's comments on our Open Question in the September 1984 issue of TIBS 'Is phosphofructokinase the rate limiting step of glycolysis?' were grate fully received. They are however, directed toward minor points related to the formal, theoretical treatment and they miss the main point we tried to raise, namely, that the sensitivity coefficient (control strength) for phosphofructokinase depends not only on the set of experimental conditions chosen (effectors, $\mathrm{pH}$, state of aggregation of the enzyme) but also on the type of cell and on the type of organism in question.

We would like to point out that different terms have been used by different authors when describing similar concepts pertaining to control analysis, Only recently has a standard terminology been submitted for study to the IUB committee on Nomenclature.

Three further comments:

If one ignores the value of $Z$ for an enzyme in different types of cells, a good approach to approximate its participation in the control of metabolic flux is to calculate the mass action ratio and constant independently of the cell type, the fructose 1,6-bisphosphate/fructose 6-phosphate $([\mathrm{F} 1,6 \mathrm{P} 2] /[\mathrm{F} 6 \mathrm{P}])$ ratio gives a good approximation to changes in the ratio for phosphofructokinase (PFK).

We gave the $\mathrm{Km}$ value for $\mathrm{F} 1,6 \mathrm{P} 2$ for mammalian aldolase that is lower than the one for yeast aldolase. At any rate, the fact that the F1, 6P 2 concentration in glucose-consuming yeast is $\sim 4 \mathrm{mM}$ gives a [substrate] $/ \mathrm{Km}$ ratio higher than 10 and thus aldolase should be saturated by its substrate.

Calculations that leave glyceraldehyde-3-phosphate dehydrogenase (and perhaps other enzymes down the pathway) out of the glycolytic flux control do not explain the accumulation of F1,6P 2 observed in some cell types. 
We acknowledge that classification of PFK types according to $[\mathrm{F} 6 \mathrm{P}] /[\mathrm{F} 1,6 \mathrm{P} 2]$ ratios is an oversimplification and that the solution to the problem could be to calculate $Z$ values for PFK from different sources under standardized conditions. This cannot be done with the data found in the literature but would be a very interesting project for future experimental work.

As far as Dr Porteous' letter is concerned, we must emphasize that we did not purport to use the concepts of Kacser and Burns except as an introduction to call attention to our main point.

It would be improper here to attempt a criticism of that theory, but it is important to point out that if one uses it without due attention to the physiological context in which enzymes work, one must overlook the facts that: metabolic pathways are seldom linear; there are enzymes that do not exhibit Michaelis kinetics (some of which are precisely those claimed to control a pathway, e.g. PFK and pyruvate kinase); the precise available concentrations of free substrates in a cell are not always known and in fact, for some, it is only a fraction of the total concentration, e.g. F1,6P2; in a cell, both substrates and products are continuously available to the enzyme; effectors change the conformation and thus the activity of certain enzymes; and there are protein-protein interactions at physiological enzyme concentrations that considerably modify the in vitro kinetics. As expected, all this is well understood by Dr Racker. 